\title{
The Recognition of Maize seeds Based on Multi-scale Feature Fusion and Extreme Learning Machine
}

\author{
Mingzhi $\mathrm{Du}^{1,2}$, Xiao $\mathrm{Ke}^{1,2^{*}}$,Mingke Zhou ${ }^{1,2}$ \\ ${ }^{1}$ College of Mathematics and Computer Science, Fuzhou University, Fuzhou, 350116, China \\ ${ }^{2}$ Fujian Provincial Key Laboratory of Networking Computing and Intelligent Information Processing \\ (Fuzhou University), Fuzhou, 350116, China \\ *Email: kex@fzu.edu.cn
}

Keywords: extreme learning machine, feature fusion, object recognition

\begin{abstract}
In recognizing traditional crops seeds like maize seeds, we usually use electrophoresis assay method, fluorescence scanning method and chemical assay method. These methods are destructive methods. They take a long time to detect and are demanding of professional background knowledge and hardware conditions etc. What's more, these methods, based on BP neural network and support vector machine (SVM) while taking a long time to detect are less accurate in process of classification. In this paper, based on the computer vision technology, we proposed a new method for the classification of maize seeds, a method based on multi-scale feature fusion and extreme learning machine. First, we extract the multi-scale fusion feature of maize seeds. Second, based on extreme learning machine, we construct the classifier model of maize seed. Third, because of the window of image in the case of multi-scale detection has the problem of capturing the same object seed with many overlapping windows, we put forward a kind of window fusion algorithm to solve it. The simulation results show that: The method is able to identify the maize seeds accurately. Using this method the accuracy of classification of maize seeds can reached $97.66 \%$ and the error rate is less than $0.1 \%$. Compared with the traditional methods, the method we proposed can improve the speed of detection and the accuracy of classification, and has no strict hardware requirements.
\end{abstract}

\section{Introduction}

Seeds are the most basic means of production in agricultural industry, it is the foundation of all agricultural production. Accurate and rapid identification of the seeds has great instructive significance in agricultural production. How to identify crop seed in a nondestructive, rapid way is an important part of the researches in this field. In recent years, with the rapid development of machine learning, many scholars at home and abroad have applied machine learning in the automatic detection of seed used in agricultural production [1]. When using machine vision to identify the seeds, in general, the first step is extracting the color [2] and shape feature of seed and others features, and then identify them through classifiers, such as Bayesian classifier, the BP neural network and support vector machine (SVM) classifier.

For example: Shuqin Yang, etc, all of them applied BP neural network in the identification of maize varieties [3]. After analyzing the characters of grain shape and extracting the shape, size, color and other related structural features of corn grain. They used these features in classification and recognition conducted by the BP neural network, but the training time is too long and the accuracy is low. Hongyong Wang, etc, combined genetic algorithm and support vector machine (SVM) in recognizing maize seeds [4]. Using genetic algorithm to extract the corn seed's feature information , transfer it to training support vector machine (SVM) classifier, so as to identify maize varieties. Their comprehensive recognition rate is higher, but this method takes up much time and space to detect. In addition, Huang, etc[5], also used a spectral image characteristics method to study single seed identification, they have gained a high recognition accuracy in the corn seed testing set data. But studies [5][6] based on single feature recognition model can only identify a limited number of seed and require specialized hardware devices.

The traditional seed identification methods has a slow detection speed and a long training time. Its 
identification accuracy is low and its high demand for hardware equipment are also a problems, so we proposes a multi-scale fusion feature extraction and seed identification method based on the extreme learning machine. This method can realize accurate identification of the corn seed. From the results of tests on the popular seed varieties such as the Aoyu311, Huayu986, Xinyu35, etc, we find that the test accuracy can reach $97.66 \%$ and the error is within $0.1 \%$. Compared with traditional methods, the proposed method improved detecting speed, the training speed and identification precision and with no additional high demand of hardware equipment.

\section{The Corn Seed Recognition Framework}

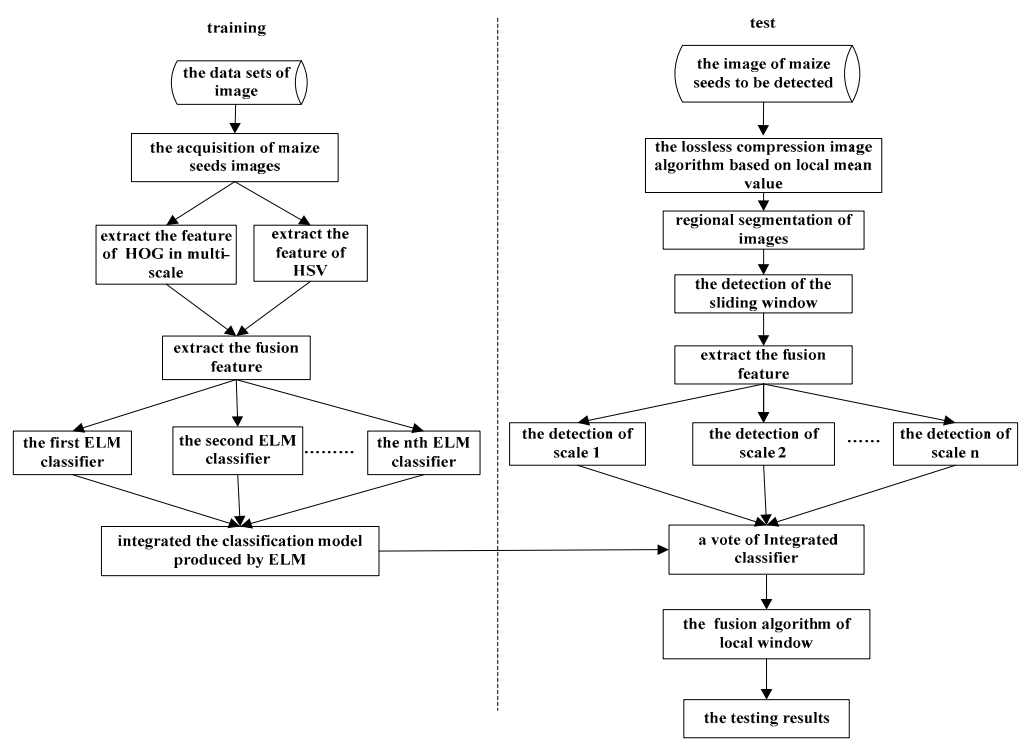

Fig.1 Overall frame chart of the algorithm

At first, we extract the HOG and multi-scale based HSV features of collected corn seeds. Second, use the extreme learning machine to train the collected feature information of corn seeds and generate classification model. Finally, we use the trained classifier to classify the features. We used a voting system to achieve final recognition results. In the last section of this article, we put forward a kind of local window fusion that based on fuzzy clustering algorithm to optimize our result.

\section{Multi-scale feature fusion}

The main idea is to calculate the distribution of gradient direction histogram of different scales. As shown in figure 2, after segmenting seminal image level-by-level, we divide each level into a few local areas, and then we use gradient direction histogram of blocks of each level to create a multi-scale gradient direction histogram in the order showed in Fig.2.

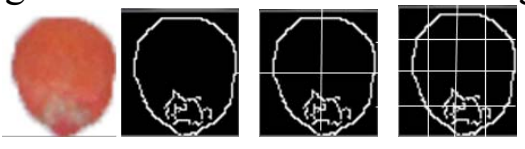

$L=0 \quad L=1 \quad L=2$

Fig.2 The Multi-scale feature extraction of Hog of maize seed

Through cascading all edge gradient features of different level and then normalizing the cascade feature, the multi-scale feature achieved. The method, by fusing space feature and local feature, has a strong resilient against inaccuracy result form rotation and noisy. Our Sliding window mechanism is shown in figure 3 : 


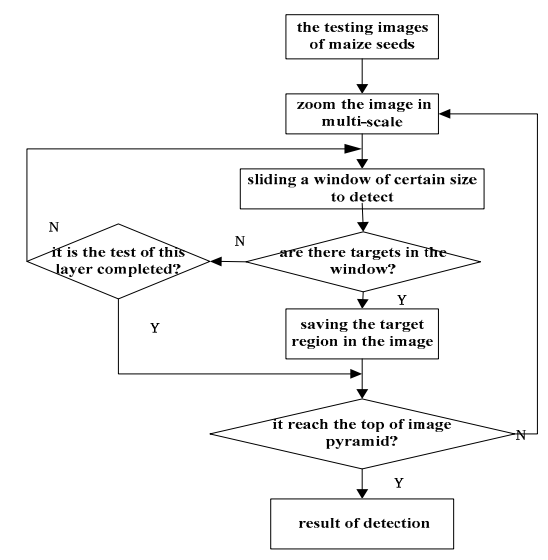

Fig.3 The Multi-scale detection of sliding window

\section{Extreme learning machine and integrated classification model}

ELM is a simple and effective single hidden layer feed forward neural network learning algorithm [7]. Compared with traditional neural network learning algorithm, ELM don't required a lot of manually set training parameters, it only require setting the number of hidden layer node. And not adjustment in the offset value of hidden unit and input weight is need. In addition, the traditional algorithms of neural networks can easily lead to local optimal solution or get into back and forth iteration oscillation. On the contrary, the external weight of ELM's network is obtained by minimizing least square solution of squared-error loss function, it's the only optimal solution. ELM can greatly reduce the time consumption in the regulation of network parameters, also it learns fast and has great generalization ability. And In our research, the number of maize seed to be identified is huge and the seeds are closely located, and applying ELM can greatly increase the efficiency in seed recognition.

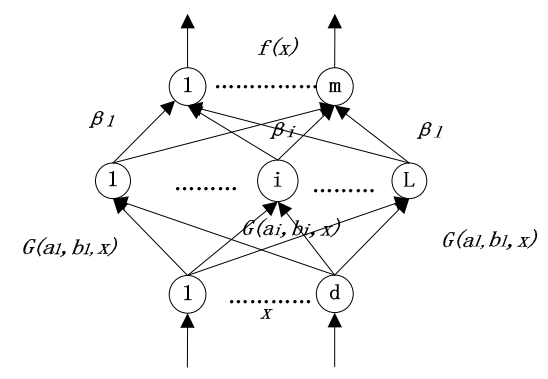

Fig.4 Basic model of extreme learning machine

In order to improve the accuracy of classification, we construct some different classifier for voting. Ensemble learning, such as algorithms Bagging and Adaboost, can improve the accuracy of classification. The main idea of Bagging is extracting recovery sample from the training data ,then constructing for every base classifier a training set with a contain that is slightly different from the original one. Randomness is the key factor in deciding whether Bagging can improve the accuracy of classification or predict. Bagging can improve the accuracy of prediction of random learning algorithm. The new model in machine learning -- ELM, has parameter randomness, so we can use Bagging in ensemble learning to improve the classification precision.

\section{Local window fusion algorithm}

As it mentioned before, we adopt the sliding window mechanism to detect corn seed in images. But sliding window in multi-scale environment could lead to overlapping of the windows; as a result, multiple sizes of window in the same image could turn out to be marking the same object of seed. Therefore, to improve the accuracy of recognition, we must fuse the multiple overlapping windows marking out the same seed. In computer vision-based image processing, fuzzy clustering 
is widely used in image retrieval [8][9], image segmentation, image edge detection, image enhancement, image compression, etc. Although fuzzy clustering is widely used, it have not been used in detecting window fusion problems. Based on fuzzy clustering algorithm, we put forward a kind of local window fusion.

The basic idea is treat the window as different discrete points in the two-dimensional space, and then local window fusion can be seen as the clustering of these discrete points. By calculating the clustering center, we can get the best fusion window position.

Algorithm 1: window fusion algorithm proposed in this paper

input: The coordinates of the candidate maize seeds $x_{i}=\left\{x_{i}, y_{i t}, w_{i}, h_{i}\right\}_{i=1}^{n}$

output: $\quad \operatorname{set}[i]_{i=1}^{k} \quad / /$ Where $\mathrm{k}$ represents the number of sets of Windows after carry

on the fusion algorithm.

Initialization:

1: Sorted the window judged by the classifier as windows marking out the candidate seeds according to there size .

2: Determine the punishment factor: $p$

start:

1) for $\mathrm{i}=0: n-1$

2) for $\mathrm{j}=0: 1$

3) compute $R_{n \times 2}$; // calculate fuzzy matrix according the maximum normalization method

4) end for

5) end for

6) for $\mathrm{i}, \mathrm{j}=0: \mathrm{n}-1$;

7) if $\mathrm{i}=\mathrm{j}$ then $r_{i j}=1$;

8) else $r_{i j}=1-\frac{p}{e^{\frac{\text { overlap }\left(x_{x}, x_{j}\right)}{\sqrt{\left(x_{x}-x_{\mu}\right)^{2}+\left(x_{j}-x_{j}\right)^{2}}}}}$; // Calculating the fuzzy similarity matrix

9) end for

10) for $\mathrm{i}=1: \max ; / / \max$ is a large integer

11) if $R^{i} \circ R^{i}=R^{i}$ continue;

12) else $\mathrm{i}=\mathrm{i}+1$ compute $R^{i} \circ R^{i}$;

13) end for

14) output $R^{*}$; // use the method of square self consistency to compute equivalence matrix $R^{*}$

15) Sorted according to the score of matrix $R^{*}$, randomly selected a appropriate $\lambda$, and dividing setunion []$_{i=1}^{k}$ according to it ;

16) using weighted average method to process each type of window ,output $\operatorname{set}[i]_{=1}^{k}$;

The algorithm description of this paper is as follows:

1) Assume $U=\left\{x_{1}, x_{2} \cdots x_{n}\right\}$ is a set of detection window awaiting classification, here $x_{i}=\left\{x_{i t}, y_{i t}, w_{i}, h_{i}\right\}$. $\left\{x_{i t}, y_{i t}\right\}$ denotes the upper left coordinate of the sliding window, $w_{i}$ denotes the wide of sliding window, $h_{i}$ denotes the height of sliding window, each element $x_{i}$ of the set has two feature $x_{i x}, x_{i y}$. here, $x_{i x}=x_{i t}+w_{i}, x_{i y}=y_{i t}+h_{i}$, normalize the fuzzy matrix to [0,1]. Here we use the maximum normalization method:

$$
x_{i j}^{\prime}=\frac{x_{i j}}{M_{j}}, M_{j}=\max \left(x_{1 j}, x_{2 j} \ldots \ldots x_{n j}\right)
$$

2) Use set $U$ to construct $R=\left(r_{i j}\right)_{n \times n}$, namely to compute element value $r_{i j}$ in the fuzzy similar matrix , $r_{i j}$ denotes similarity between window $x_{i}$ and window $x_{j}$. Here we proposes a new method of similarity calculation that specifically address the detection:

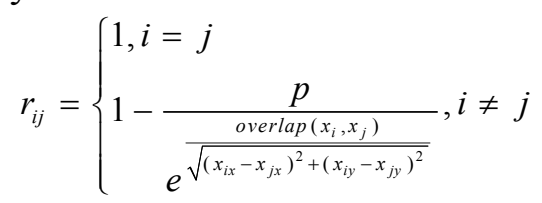

Where, $p$ is the penalty factor and is a constant that used to adjust central distance and 
overlapping area. $\operatorname{overlap}\left(x_{i}, x_{j}\right)=\operatorname{area}\left(x_{i} \cap x_{j}\right)$ denotes the overlapping area, so we have:

$$
\begin{gathered}
\operatorname{area}\left(x_{i} \cap x_{j}\right)=d w \times d h \\
d w=\max \left(\left(\min \left(x_{i x}, x_{j x}\right)-\max \left(x_{i t}, x_{j t}\right)\right), 0\right) \\
d h=\max \left(\left(\min \left(x_{i y}, x_{j y}\right)-\max \left(y_{i t}, y_{j t}\right)\right), 0\right)
\end{gathered}
$$

In this paper, we defined the similarity function as the overlapping area and the central distance between the field windows, these two factors are the key factor deciding that weather the field can

be fusion locally. Here, $\frac{\operatorname{overlap}\left(x_{i}, x_{j}\right)}{\sqrt{\left(x_{i x}-x_{j x}\right)^{2}+\left(x_{i j}-x_{j y}\right)^{2}}}$ is called overlapping area density, namely the ratio between the overlapping area of field window and central distance, When the overlapping area is large and the central distance is relatively small, the degree of similarity between windows will be higher, and therefore the windows are more likely to cluster into a same class.

3 ) According to the obtained fuzzy similarity matrix, the transfer closure of fuzzy matrix can be calculated. The fuzzy equivalent matrix $R^{*}$ of that used in the detection of windows can be obtained by the method of square self consistency.

4) Through setting optimal value of $\lambda$, we can get the calculate the quotient set $U / R_{\lambda}^{*}$ of $\lambda$-cut equivalence matrix $R_{\lambda}^{*}$ corresponding to $U$, we can get the clustering results of all the candidate windows in the image. In this paper, we only need to give a $\lambda$ value for fusion to get the classification results, the value of $\lambda$ can be determined by sifting result of experiments.

5 ) By the above algorithm, we can get the set of windows in each clustering result. First we calculate the central position of the set that includes those multiple windows. Second we calculate the width and height of the window according to the weighted average. In this paper, we use the Gauss density distribution method. The more the window is close to the window center, the larger weight, and last, through this process we can obtain the final position of window.

\section{Experimentation}

The samples to be tested consist of 130000 maize seeds. Their varieties are Aoyu311, Huayu986 and Xinyu35. Here, we use the 5 - a fold Cross Validation method and the comprehensive index to assess the result, and calculate the recall rate and accuracy. In the data set, the number of positive sample is 2600 and the number of negative sample is 2600 .

$$
\begin{aligned}
R & =\frac{Y}{T} \\
P & =\frac{Y}{Y+W} \\
F & =\frac{2 * P^{*} R}{P+R}
\end{aligned}
$$

Where Y presents the number of seeds which identified correctly, $\mathrm{T}$ presents the total number of seeds, $\mathrm{W}$ presents the number of seeds which identified wrong. The evaluation index in this paper is $\mathrm{R}, \mathrm{P}$ and $\mathrm{F}$.

Figure 5 shows the comparison of the fusion algorithm of the seed of huayu 986 before and after the merging. Figure 6 shows the comparison of the fusion algorithm of the xinyu 35 seed before and after the merging. It shows that the fuzzy clustering algorithm-based local window fusion we used can greatly optimize the recognition results.
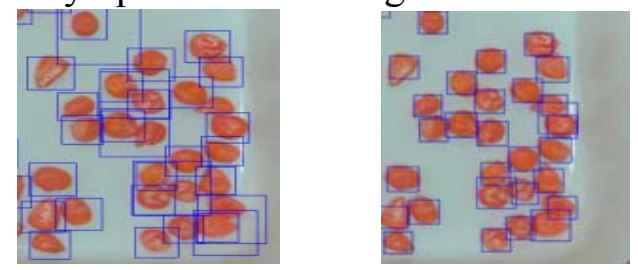
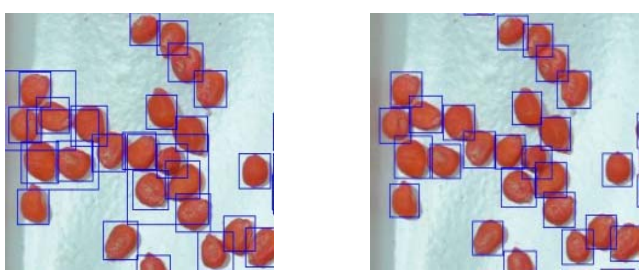

Fig.5 The comparison of the fusion algorithm of Fig.6 The comparison of the fusion algorithm of the seed of huayu 986 before and after the seed of xinyu 35 before and after

In the experiment we use extreme learning machine (hereafter ELM) as the recognition classifier and multithreaded ensemble as learning method. The result as it shown in Figure 7, it that the 
detection efficiency has been remarkable improved. The mean detection time is much shorter than that of the traditional classification and recognition method based on BP neural network and SVM. From Figure 8 we can find that, by extracting the features of maize seeds through integrating multi-scale features, the seed information can be accurately describe with the recognition accuracy amounting to 97.8 percent on average. It is a significant improvement compared with the above-mentioned traditional method based on BP neural network or SVM.

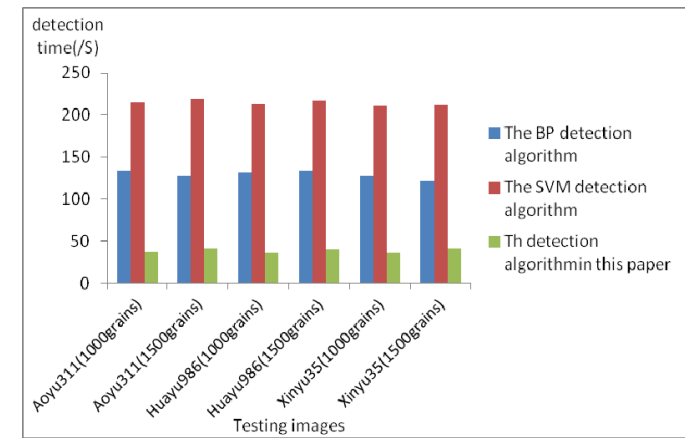

Fig.7 The comparison of the detection time

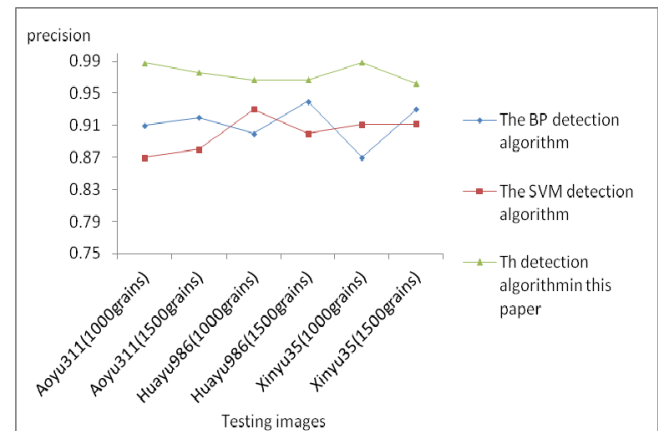

Fig.8 The comparison of accuracy based on BP、SVM and the algorithm in this paper

Form Table 1, we find that the identification algorithm we proposed have good robustness in detecting the features of light, background of seeds, seed scale, seed shape. Different from the traditional method, the algorithm is quick in training with high recognition accuracy. It also have strong generalization ability. Moreover, when the density of the seed in the dataset increases, it still can give satisfactory results .

Tab.1 The final data of experiments

\begin{tabular}{|c|c|c|c|c|c|}
\hline Test images & $\begin{array}{c}\text { consumption of time } \\
(\mathrm{S})\end{array}$ & $\begin{array}{c}\text { consumption of memory } \\
\text { (M) }\end{array}$ & $\begin{array}{c}\text { accuracy } \\
(\mathrm{P} \%)\end{array}$ & $\begin{array}{r}\text { recall rate } \\
(\mathrm{R} \%)\end{array}$ & $\begin{array}{c}\text { comprehensive index } \\
(\mathrm{F} \%)\end{array}$ \\
\hline Aoyu311(100 & grains) & 3360 & 98.83 & 97.24 & 98.02 \\
\hline Aоуu311(150 & grains) & 3365 & 97.62 & 96.19 & 96.89 \\
\hline Huayu986(10 & grains) & 3369 & 96.77 & 97.88 & 97.32 \\
\hline Huayu986(15 & grains) & 3366 & 96.74 & 97.22 & 96.48 \\
\hline Xinyu35(100 & grains) & 3366 & 98.88 & 97.92 & 98.09 \\
\hline Xinyu35(150 & grains) & 3367 & 96.23 & 95.90 & 96.06 \\
\hline
\end{tabular}

\section{Conclusion}

In this paper, we present a recognition algorithm of maize seed based on ELM and multi-scale feature fusion. Based on this recognition algorithm we proposed a local window fusion algorithm to improve the recognition rate which adds something new to study of machine learning applied in seed identification. Experimental results show that the method we proposed has some practical and theoretical research value. Overall, the method we use less time, and is more effective. It should also be noted that the algorithm we propose could be father improved. We notice that there are still deficiencies in the identification result generated by our method. For example, sometimes target seed are omitted or be counted again. This is the common problem on the identification test based on machine learning. How to further improve the seed recognition accuracy and less the time and memory usage while limited computer resources usage in a reasonable level is the focus our further research.

\section{Acknowledgement}

This work is partially supported by the National Natural Science Foundation of China under Grants No. 61103175, the Natural Science Foundation of Fujian Province under Grant No. 2013J05088, the Fujian Province High School Science Fund for Distinguished Young Scholars under Grand No.JA12016. 


\section{References}

[1] Sun Kaiqiong, Chen Zhen, Jiang Shaofeng. Local morphology fitting active contour for automatic vascular segmentation[J]. IEEE Transactions on Biomedical Engineering, 2012, 59(2):464 473.

[2] Yan Xiao-mei, Liu Shuang-xi, Zhang Chun-qing, et al.. Purity identification of maize seed based on color characteristics [J]. Transactions of the CASE, 2010, 26(S1):46 50.

[3] Yang Shu-qin, Ning Jif-eng, He Dong-jian. Identification of corn breeds by BP netural network [J]. Journal of Northwest Sci-Tech University of Agriculture and Forestry(Natural Science Edition),2004,32:162 164.

[4] Wang Hong-yong, Hou Hui-fang, Liu Su-hua . Maize seed recognition based on genetic algorithm and multi-class SVM [J].Computer Engineering And Applications, 2008, 44(18):221 223.(in Chinese).

[5] Huang Min, Zhu Xiao, Zhu Qi-bing, et al.. Hyperspectral image classification of maize seeds based on active contour model [J]. Journal of Data Acquisition and Processing, 2013, 28(3): 289 293.

[6] Huang Min, Zhu Xiao, Zhu Qi-bing, et al.. Morphological characteristics of maize seed extraction and identification based on the hyperspectral image [J]. Acta Photonica Sinica, 2012, 41(7): 868 873.

[7] Huang G B, Zhu Q Y, Siew C K. Extreme learning machine: theory and applications[J]. Neurocomputing,2006,70(1/3):489 501.

[8] Li Lan, Liu Yang, Ma Zhen, et al..Image retrieval method on image features of fuzzy clustering [J]. Journal of Tsinghua University: Science and Technology, 2014,54(7):929 934.(in Chinese)

[9] K.Haridas, Antony Selvadoss Thanamani. An Efficient Image Clustering and Content Based Image Retrieval Using Fuzzy K Means Clustering Algorithm[J].International Review on Computers and Software,2014,9(1):147 153. 